\title{
Bone-marrow-derived cells could cause gastric preneoplasia in chronic Helicobacter pylori infection
}

Bone-marrow-derived cells (BMDCs) accumulate in the gastric epithelium as a result of Helicobacter pylori infection and could contribute to tumor development, according to French scientists. H. pylori virulence factors stimulate chronic inflammation that induces recruitment, differentiation and transformation of BMDCs-potentially initiating cancer.

BMDCs expressing green fluorescent protein were transplanted from male donor mice into $H$. pylori infected C57BL/6J female mice. Infection led to inflammation, hyperplasia, metaplasia and dysplasia. After 1 year, 50-90\% of gastric glands from infected female mice contained male BMDCs and $22 \%$ of dysplastic lesions induced by $H$. pylori were composed of BMDC-containing glands. "Although BMDCs are not the most frequent origin of gastric dysplasia, cancer might develop from these cells," explain Christine Varon and Francis Mégraud of INSERM, France.
The traditional view that cancer cells arise through transformation of local epithelial or progenitor cells was challenged in 2004 by a mouse study using the feline pathogen, Helicobacter felis, where BMDCs were found in dysplastic lesions. Varon and Mégraud's work corroborates those results and shows the accumulation of BMDCs following infection with the known human pathogen, $H$. pylori. By

Bone-marrow-derived cells (brown) in metaplastic epithelial glands in the stomach of an $\mathrm{H}$. pylori infected mouse.

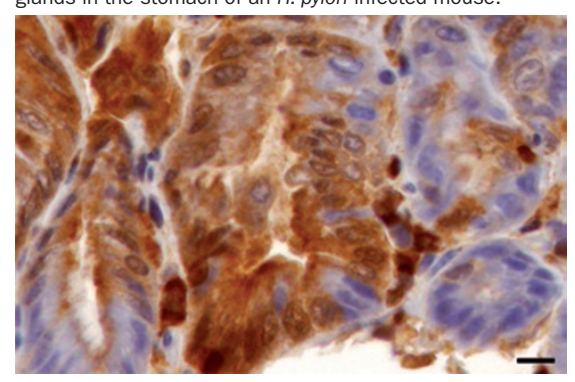

using multiple strains of the bacteria with different proinflammatory virulence factors, they show that chronic inflammation drives the recruitment of BMDCs to the site of infection and that undiscovered virulence factors could contribute. "It is interesting that known pathogenic factors of $H$. pylori might not be involved," say Varon and Mégraud.

The researchers are now trying to characterize these BMDCs and elucidate both the chemotactic signals that drive their recruitment and the virulence factors that influence this process. "Cancer stem cells could originate from BMDCs in some tumors," Varon and Mégraud conclude.

Andy McLarnon

Original article Varon, C. et al. Helicobacter pylori infection recruits bone-marrow-derived cells that participate in gastric preneoplasia in mice. Gastroenterology doi:10.1053/j.gastro.2011.10.036 